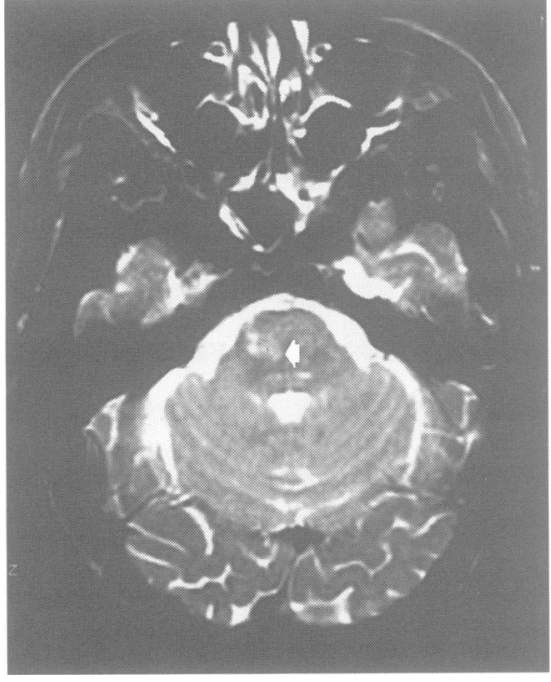

Figure 1 Axial T2 weighted MRI of mid-pons showing infarct in the right lateral basis pontis extending medially.

a blood pressure of 120/72. He had a left central facial weakness and a mild left hemiparesis affecting the arm and leg equally. Incoordination of the left upper extremity was present on finger to nose testing and bilateral heel-knee-shin ataxia was present, worse on the left. The muscle stretch reflexes were normal and the plantar reflexes were flexor bilaterally. Brain MRI disclosed an area of increased signal intensity involving both the paramedian mid-pons and extending laterally in the caudal third of the pons (fig 1).

It is unclear why patients with ataxic hemiparesis usually have ataxia contralateral to the lesion rather than bilateral limb ataxia. It has been suggested that the pon-

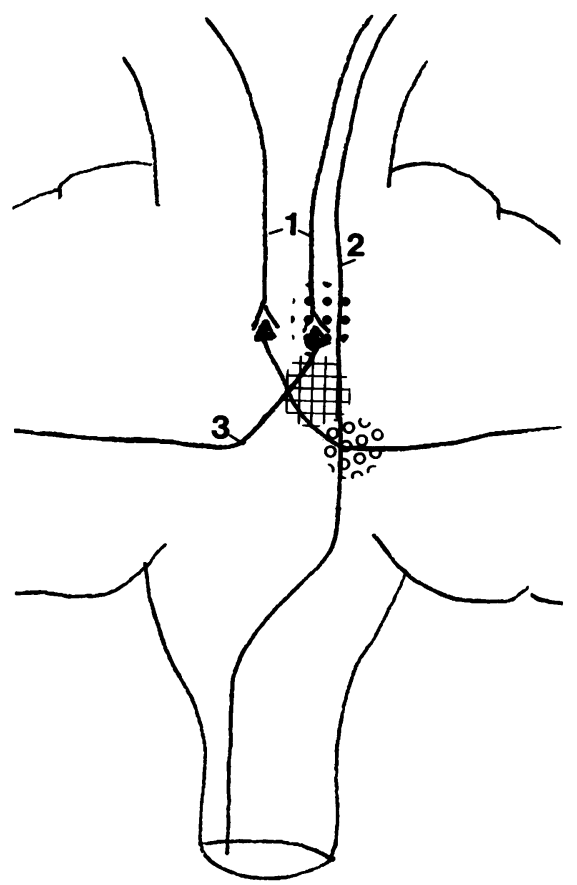

Figure 2 Diagram of coronal section through brainstem showing corticopontine fibres (1), corticospinal tract (2), and pontocerebellar fibres (3). The site of a pontine lesion causing ataxic hemiparesis as described by Fisher is shown in bold dots, the site of a lesion causing ipsilateral ataxia ${ }^{4}$ is shown in open dots, and the site of the lesion in our patient is shown in cross hatching. tine nuclei are more vulnerable to ischaemia than the transverse pontocerebellar fibres crossing from the contralateral side but there is no histological evidence for this. ${ }^{3}$ Huang and Chang $^{2}$ suggested that the crossing transverse pontocerebellar fibres take an oblique downward course to the contralateral middle cerebellar peduncle and in this way a more rostrally placed pontine lesion may involve only the corticopontine fibres and pontine nuclei ipsilaterally, but miss the more caudally placed crossing pontocerebellar fibres. A more caudolateral lesion would be expected to give rise to ipsilateral ataxia (fig 2). Our case supports this suggestion, as the lesion extended more laterally and caudally than previously described pontine infarcts associated with ataxic hemiparesis. The more caudolateral part of the infarct in our patient may have damaged the crossed pontocerebellar fibres and have resulted in the leg ataxia ipsilateral to the infarct, whereas the more medial and rostral part may have given a contralateral ataxic hemiparesis (fig 2). This suggestion is supported by Fisher's report of a lower lateral pontine infarct ${ }^{4}$ and Fisher and Tapia's report of a lateral medullary infarct extending into the lower lateral pons. ${ }^{5}$ Both these infarcts gave rise to ataxia only ipsilateral to the lesion.

$$
\begin{array}{r}
\text { SHERRY WITHIAM-LEITCH } \\
\text { PATRICK PULLICINO } \\
\text { Department of Neurology, State University of } \\
\text { New York at Buffalo, } \\
\text { Buffalo General Hospital, 100 High Street, }
\end{array}
$$
Buffalo NY 14203, USA

Correspondence to: Dr S Withiam-Leitch

1 Fisher CM. Ataxic hemiparesis. A pathologic study. Arch Neurol 1978;35:126-8.

2 Huang $\mathrm{CY}$, Chang KH. Pontine ataxic hemiparesis, a lateral penetrator syndrome? Neurol Neurosurg Psychiatry 1984;47:1046-7.

3 Nabatame H, Fukuyama H, Akiguchi I Kameyama M, Nishimura K, Torizuka K Pontine ataxic hemiparesis studied by a high resolution magnetic resonance imaging system. Ann Neurol 1987;21:204-7.

4 Fisher CM. Lacunar infarct of the tegmentum of the lower lateral pons. Arch Neurol 1989; 46:566-7.

5 Fisher CM, Tapia J. Lateral medullary infarction extending to the lower pons. I Neurol extending to the lower pons.
Neurosurg Psychiatry 1987;50:620-4.

\section{Autoimmune chronic active hepatitis and polymyositis in a patient with myasthenia gravis and thymoma}

Myasthenia gravis is known to occur with other autoimmune disease. It is rarely associated with polymyositis, ${ }^{1}$ and very exceptionally with autoimmune chronic active hepatitis. ${ }^{2}$ We describe a patient with generalised myasthenia gravis, cortical thymoma, polymyositis, and autoimmune chronic active hepatitis.

A 25 year old Chinese woman presented with intermittent weakness of the limbs for one month with no diplopia, speech disturbance, or bulbar symptoms. She had mild ptosis of her left eye which increased progressively with maintained upward gaze, and proximal limb muscle weakness. The rest of the examination was normal. She had a positive edrophonium test, and serum antiacetylcholine receptor and antistriated muscle antibody titres. Chest radiography disclosed a mediastinal mass which was subsequently confirmed by CT to be a thymoma measuring $4.2 \mathrm{~cm}$ (anteroposterior) $\times 3.1 \mathrm{~cm}$ (width) in the left lobe of the thy- mus. Serial serum muscle enzymes were raised. A repetitive stimulation test on three muscles showed a more than $15 \%$ decrement with postexercise exhaustion. An EMG showed abundant fibrillations, positive sharp waves and insertional irritability, and short, small polyphasic motor units. Biopsy of her deltoid muscle showed perivascular infiltration of lymphocytes with phagocytosis and variable numbers of angulated atrophic fibres.

Incidentally she was found to have hepatitis. During the initial stay in hospital, a liver function test gave albumin $45 \mathrm{~g} / \mathrm{l}$, globulin $37 \mathrm{~g} / \mathrm{l}$, alkaline phosphatase $106 \mathrm{U} / \mathrm{l}$, bilirubin $21 \mathrm{umol} / 1$, alanine aminotransferase $1158 \mathrm{U} / 1$, and aspartate aminotransferase $943 \mathrm{U} / 1$. Viral markers of hepatitis A, B, and $C$ were all negative, whereas antinuclear factor and antismooth muscle antibody were positive. Her HLA antigens were A33, B17, and DR3. Liver biopsy showed a picture of active chronic hepatitis with enlarged portal tracts and a pronounced mononuclear cell infiltrate which spilt across the limiting plate. There were foci of piecemeal necroses and the hepatocytes often displayed ballooning degeneration. Staining for hepatitis B was negative.

Her muscle weakness responded promptly but not completely to pyridostigmine ( $60 \mathrm{mg}$ four times daily), and after the liver biopsy, she was treated with prednisolone (55 mg daily) and azathioprine $(50 \mathrm{mg}$ ) daily. Three weeks after, she developed ophthalmic herpes, which was treated with acyclovir.

She continued to improve both in muscle power and liver function. Two months after treatment with prednisolone and azathioprine, the liver enzymes were considerably decreased-alanine aminotransferase $39 \mathrm{U} / 1$ and aspartate aminotransferase $38 \mathrm{U} / 1$. Thymectomy was then performed and the postoperative course was uneventful. Histology confirmed that the thymoma was encapsulated, non-invasive, and of cortical type.

This is the first report of the combination of generalised myasthenia gravis with thymoma, polymyositis, and autoimmune chronic active hepatitis. Fifty per cent of patients with chronic active hepatitis will die of liver failure within five years if no treatment is given. Although the prognosis can be considerably improved with steroid and azathioprine treatment, most patients develop cirrhosis. ${ }^{3}$

The susceptibility of the patient to generalised myasthenia gravis, autoimmune chronic active hepatitis, and polymyositis seems to be related to HLA DR3. ${ }^{4}$ There is probably an interplay of genetic and environmental factors in the occurrence of these diseases. Interestingly, the association of polymyositis and HLA DR3 is claimed to be related to the coexistent presence of antibodies to histidyl tRNA synthetase (Jo-1). In this regard the appearance of such autoantibodies in polymyositis is reported to be most uncommon ${ }^{4}$ and was absent in the patient reported herein. Despite the coexistence of the diseases, a good response was obtained with steroid combined with azathioprine and thymectomy.

KWAI FU KO TINNY HO Department of Medicine Department of Pathology, Department of Pathology,
University of Hong Kong, Queen Mary Hospital, Pokfulam Road, Hong Kong 
Correspondence to: Dr Kwai Fu Ko, Block 5, Flat D, 19/F, City Garden, 233 Electric Road, North Point, Hong Kong.

1 John TR, Crowleg WJ, Miller JQ, et al. The syndrome of myasthenia and polymyositis with comments on therapy. Ann NY Acad Sci 1971;183:64-71

2 Kikuchi H, Hayashi N, Nakajima T, et al. A case report of autoimmune hepatitis with myasthenia gravis. Nippon Shokakibyo Gakkai Zasshi 1988;85:2261-4

3 Kirk AP, Jain S, Pocock S, et al. Late results of Royal Free Hospital controlled trial of prednisolone therapy in hepatitis B surface antigen-negative chronic active hepatitis. Gut 1980;21:78-83.

4 Ehrenstein MR, Snaith ML, Isenberg DA. Idiopathic myositis: a rheumatological view. Ann Rheum Dis 1992;51:41-4.

5 Garlepp M J. Immunogenetics of inflammatory myopathies. Bailliere's Clinical Neurology 1993;2:579-97.

\section{Transient monocular blindness}

The term amaurosis fugax, which means "fleeting blindness", has come to be associated with transient monocular blindness due to emboli reaching the retinal circulation from the carotid vessels or from the heart. As amaurosis fugax may precede a stroke it is usually viewed in a neurological context. Transient monocular blindness, however, is not solely caused by emboli. It has many causes, including migraine, intracranial hypertension, and malignant hypertension and it is, therefore, important to make an accurate diagnosis. We describe a common ophthalmic condition, intermittent angle closure glaucoma, giving rise to transient monocular blindness, so emphasising the need to be aware of such diagnoses.

A 66 year old woman presented with a 12 month history of repeated episodes of transient loss of vision in her right eye. The episodes were precipitated by reading, writing, and watching television for a variable time. They came on suddenly and she described a film descending over her right eye leading to complete loss of vision. The episodes lasted from three minutes to several hours. On occasions there was an associated ache over the right side of the forehead. She had no positive visual symptomatology during an attack and there was no family history of migraine. She smoked six cigarettes a day.

She had a history of palpitations and blackouts for which she attended a cardiologist. An ECG and an exercise stress test were normal; however, a 24 hour Holter monitor had disclosed frequent ventricular extrasytoles and an episode of atrial fibrillation. She was treated with a $\beta$ blocker and subsequently amiodarone, but this was discontinued by the patient. Neurological examination was normal, as were carotid duplex scans, brain CT, and an echocardiogram. A provisional diagnosis of amaurosis fugax was made and she was started on $75 \mathrm{mg}$ aspirin a day. In view of the specific precipitating factors of her visual loss, she was referred for a neuro-ophthalmic opinion.

Initial neuro-ophthalmic assessment was normal. To assist in reaching a diagnosis it was decided to try to precipitate an attack. After reading intermittently over a period of four hours she reported loss of vision in her right eye. Slit lamp examination showed pronounced corneal oedema on the right, a poorly reacting semidilated pupil and a shallow anterior chamber. Her intraocular pressures were $50 \mathrm{~mm} \mathrm{Hg}$ on the right and
$18 \mathrm{~mm} \mathrm{Hg}$ on the left. Pulsation of the central retinal artery was noted on the right.

A diagnosis of intermittent angle closure glaucoma was made. After initial medical treatment to constrict the pupil and lower the intraocular pressure, nd-YAG laser iridotomies were performed. Subsequent gonioscopy confirmed a narrow drainage angle and refraction disclosed a moderate degree of hypermetropia which may be associated with a shallow anterior chamber and narrow drainage angle. At a review appointment three months later, the patient reported that since the iridotomies she had had no further episodes of visual disturbance.

The first report that transient monocular blindness could precede contralateral hemiplegia was by Miller Fisher in $1952 .{ }^{1} \mathrm{He}$ stated that "Blindness is usually complete in the affected eye, although at times the defect is limited to one sector. The frequency of attacks varies from several a day to a few each year. Symptoms last for years or may disappear completely after a few months...The blindness most commonly comes on as though a blind were being lowered or raised, and vision returns from the opposite direction... The attacks last from a minute or so up to seven minutes or more." This led to the awareness that transient monocular blindness may warn of an impending stroke and the need to institute preventative measures.

Our patient had many of the features reported by Miller Fisher. She also had several cardiovascular risk factors, being a smoker and having a history of cardiac arrhythmias. She had features which suggested an alternative diagnosis, however. In particular the specific precipitating factors were atypical. The duration of attacks, which on occasions lasted several hours, was unusual, although attacks of amaurosis fugax of up to 24 hours have been reported. ${ }^{2}$

A diagnosis of angle closure glaucoma was not originally suspected, as on direct questioning the patient neither reported seeing haloes, nor having any visual loss associated with poor lighting conditions. Angle closure glaucoma is usually associated with severe pain and injection of the globe, whereas our patient had complained only of an aching sensation and had not noted any redness of the eye.

Angle closure glaucoma has previously been reported as a cause of transient monocular visual loss. ${ }^{3}$ In the three cases reported the initial presenting diagnoses were either of amaurosis fugax or migrainous phenomena. Two of the patients described seeing haloes, however, and none of them had specific precipitating factors such as reading. Close ocular work such as reading and sewing can precipitate angle closure glaucoma. It is, however, uncommon for reading to be the sole precipitating factor.

Angle closure glaucoma can cause visual loss by various different mechanisms. The raised intraocular pressure leads to corneal clouding due to oedema and may reduce the perfusion pressure of the eye, thereby impairing blood flow to the choroid, retina, and optic disc. 4

In conclusion, although emboli are responsible for most cases of transient monocular blindness other causes should always be considered (see review by Gautier ${ }^{5}$ ), especially in the presence of atypical features such as specific precipitating factors or an unusually long duration of symptoms.
EOIN O'SULLIVAN SANDIP SHAUNAK TIMOTHY MATTHEWS CHRISTOPHER KENNARD Academic Unit of Neuroscience, Neuroscience,

Charing Cross and Westminster Medical School,
London, UK
PETER SIMCOCK

Charing Cross and Westminster Medical School,
London, UK
PETER SIMCOCK Department of Ophthalmology, Charing Cross
Hospital, London, UK

Hospital, London, UK

Correspondence to: Professor C Kennard, Academic Unit of Neuroscience, Charing Cross and Westminster Medical

1 Fisher CM. Transient monocular blindness associated with hemiplegia. Arch Ophthalmol 1952;47:167-203.

2 Parkin PJ, Kendall BE, Marshall J, McDonald WI. Amaurosis fugax: some aspects of management. 7 Neurol Neurosurg Psychiatry 1982. 45:1-6.

3 Ravits J, Seybold ME. Transient monocular loss from narrow angle glaucoma. Arch loss from narrow an

4 Best M, Blumenthal M, Futterman HA, Galin MA. Critical closure of intraocular blood MA. Critical closure of intraocular blo
vessels. Arch Ophthalmol 1969;82:385-92.

5 Gautier J-C. Clinical presentation and differential diagnosis of amaurosis fugax. In: Bernstein EF, ed. Amaurosis fugax. New York: Springer-Verlag, 1988:24-42.

Acquired bilateral opercular lesions or Foix-Chavany-Marie syndrome and eating epilepsy

The Foix-Chavany-Marie syndrome or bilateral anterior opercular syndrome (AOS) consists of lower facial and glossopharyngeal diplegia secondary to dysfunction of the rolandic opercula. It is usually seen in adults. It can result from tumour or infection but is mainly due to successive strokes involving both opercular regions. ${ }^{1}$ Children developing AOS acutely as a consequence of an acute CNS illness have been described. ${ }^{2}$

A 20 year old man had normal development until, at the age of 4 years, he developed acute, presumably viral, meningoencephalitis. This led to a prolonged stay in hospital in an intensive care unit. $\mathrm{He}$ was left with severe dysarthria, dysphasia, and bilateral labioglossopharyngeal paresis. At the age of 11 he developed partial motor seizures (involving the left facial muscles) with occasional secondary generalisation. Attacks occurred only during meals and there was no relation with any specific foods, definite times, or other specific triggering factors. He had received different antiepileptic regimens with poor results until combined treatment with carbamazepine $(600 \mathrm{mg} /$ day $)$, valproate $(1500 \mathrm{mg} /$ day $)$, and clobazam $(15 \mathrm{mg} /$ day) was initiated. He now has only one or two seizures a year, always triggered by eating.

On neurological examination his mouth was always open and he drooled continuously. He had bilateral lip, tongue, and pharyngeal weakness with dissociation of automatic and voluntary movements of the lower face (voluntary movements impaired and automatic movements preserved). Eye closure and extraocular movements were normal. Affect was normal. He had a brisk jaw jerk. Language was limited to guttural vowel sounds, but his comprehension was normal. He had a minimal left upper limb paresis and generalised hyperreflexia, more pronounced on the left. Computed tomography and MRI showed atrophic lesions involving both rolandic opercula (figure). Recordings from EEG showed normal back- 